\title{
Mathematical modeling of wildfire propagation in an agricultural land
}

\author{
Hengameh R. Dehkordi ${ }^{1}$ \\ CMCC, UFABC, \\ Santo André, Brazil
}

\begin{abstract}
Every year, the wildfires cause significantly financial damages to the agricultural sector and farmers. Therefore, presenting reliable models which quickly predict the behavior of fire is of great importance to manage and control the progress of wildfire in time. In the present work, by using Randers metric and Huygens' principle, we provide a model for the propagation of wildfire in some agricultural land in the dimension 3, while some wind is blowing across the space. Some example is provided to illustrate the results.
\end{abstract}

Keywords. Randers metric; Huygens' Principle; Wavefront; Causal structure; Analogue gravity.

\section{Introduction}

Every year, wildfires wreck crops and cause significantly financial damage to the farmers and agricultural industry. Global warming due to the heat created by wildfires and toxic gases released into the air are important issues that could not be ignored [12]. Therefore, methods which provide more reliable and accurate models to predict quickly the spread of fire are of great importance in the wildfire management strategies.

In providing the models of wildfire spread, simulators, such as Phoenix, IGNITE, Bushfire, FireMaster, FARSITE, and Prometheus have been widely applied [11]. However, a new problem arises here which is reducing the errors caused by the simulators [9]. Another method being frequently used is considering some fixed frames, such as the double ellipse, lemniskata, oval shape, and tear shape; and then applying the Huygens' principle [2,8]. The problem in this method is that the curvature of space is not taken into account. In fact, it is supposed that the space is of zero curvature. Whereas, in most of the cases in reality, the curvature of space is different from zero [10]. In other words, in this method, we confine ourselves to a numbers of fixed frames while in reality the frames could be the closed regions created by any smooth and closed curves. Because of this, the presented model based on such fixed frames has sometimes noticeable deviation form the behavior of fire.

The Randers metric is a recently applied method in the process of predicting the spread of wildfire and, generally, the propagation of waves $[1,6,10]$. In fact, this metric is a strong tool to model some real phenomena in anisotropic or inhomogeneous media [7]. By applying this metric we can provide equations of the fire locations at any time, while in other methods one finds the approximate locations of fire. By the way, to the best of our knowledge, in all of the above mentioned methods (simulators and taking fixed frames), the behaviors of wildfire and waves have been studied only for spaces of dimension 2. Very recently, some methods for the propagation of fire waves [4] and water waves [5] are provided for spaces of dimension $n$. Here, we present a model for the wildfire propagation in an agricultural land of dimension 3 .

Throughout this work, we assume that some wildfire is spreading throughout some agricultural land $M$. In fact, $M$ is a field of wheat, corn, grass and so on which is also a 3-dimensional smooth manifold; for instance it could be some open subset of $\mathbb{R}^{3}$. The fuel - that is the grass, corn, wheat and so on- has been distributed homogeneously and uniformly throughout the space, and moisture and temperature are steady everywhere. The objective is providing the model of spread from time 0 to time

\footnotetext{
${ }^{1}$ hengamehraeesi@gmail.com, hengameh.r@ufabc.edu.br
} 
$T$ while some wind is blowing across the land which remains constant at each interval $\left[t_{i}, t_{i+1}\right]$. Here, $\left\{0=t_{1}, t_{2}, \cdots, t_{n}=T\right\}$ is a partition of $[0, T]$. We suppose that the fire does not create singularities or cut loci, that is no two particles of fire meet. Also, the wind must be mild, that is the center of each spherical wavefront remains inside it. It should be mentioned that most of these conditions are normal in some agricultural land and, therefore, our cases contain several situations in reality. By the way, by taking the intersection of the provided model and the land, one finds the model of propagation on the 2 dimensional space, that is the land.

To find the model we start with some rotated ellipsoid whose diagonals and angles of rotation are determined from the experimental data and laboratory. This ellipsoid depends on the wind and remains constant as long as the wind does so. From the ellipsoid we find the equation of metric and then the equations of wildfire locations at any time $\tau$, the so-called wavefronts.

The remainder of this paper is organized as follows. Some preliminaries are given in Section 2. In Section 3, we present the models of wildfire propagation for two different cases of the wind blowing across the land. In Section 4, an example of a wildfire spreading throughout some wheat field under the presence of the wind is provided which illustrates the main results.

\section{Preliminaries}

Let $M$ be a smooth manifold, $p=\left(x_{1}, \ldots, x_{n}\right) \in M$ a point of it and $T_{p} M$ the space tangent at point $p$. Assume that $\left\{\frac{\partial}{\partial x_{i}}\right\}_{i=1}^{n}$ is the canonical basis for $T_{p} M$ and $V=\left(v_{1}, \ldots, v_{n}\right) \in T_{p} M$ a vector according to this basis. A Riemannian metric on $M$ is a smooth function $h$ that assigns to each point $p \in M$ a positive-definite inner product $h_{p}: T_{p} M \times T_{p} M \rightarrow \mathbb{R}$. The smoothness condition means $p \in M \rightarrow h_{p}\left(\frac{\partial}{\partial x_{i}}, \frac{\partial}{\partial x_{j}}\right) \in \mathbb{R}$ is smooth. Given $M$ and a smooth vector field $W$ on it such that $h(W, W)<1$, the function $F: T_{p} M \rightarrow \mathbb{R}$ defined as follows

$$
F(V)=\frac{\sqrt{h^{2}(W, V)+\lambda h(V, V)}}{\lambda}-\frac{h(W, V)}{\lambda},
$$

where $\lambda=1-h(W, W)$, is a metric called the Randers metric. The pair $(M, F)$ is called a Randers space.

Given any Randers space $(M, F)$ and some piecewise smooth curve $\gamma:[a, b] \longrightarrow M$, the length of $\gamma$ is $L[\gamma]:=\int_{a}^{b} F\left(\gamma^{\prime}(t)\right) d t$. Given any two points $p, q \in M$, the distance from $p$ to $q$ is defined as

$$
d(p, q):=\inf _{\gamma} \int_{a}^{b} F\left(\gamma^{\prime}(t)\right) d t
$$

where the infimum is taken over all piecewise smooth curves $\gamma:[a, b] \longrightarrow M$ joining $p$ to $q$. A smooth curve is called a geodesic if it is locally the shortest time path connecting any two nearby points on this curve. Given a compact subset $A \subset M$, we define the distance function $\rho: M \longrightarrow \mathbb{R}$ with $\rho(p)=d(A, p)$.

Given the Randers space $(M, F)$ and a submanifold $A \subset M$, a vector $V$ is orthogonal to $A$ with respect to $F$, denoted by $V \frac{\perp}{F} A$, if for every vector $U$ tangent to $A$ we have $g_{V}(V, U)=0$. Here, $g_{V}$ is the positive-definite symmetric bilinear form associated to $F$ defined as

$$
g_{V}\left(V_{2}, V_{3}\right):=\frac{1}{2}\left(\frac{\partial^{2}}{\partial t \partial s} F^{2}\left(V+t V_{2}+s V_{3}\right)\right)_{s=t=0},
$$

where $V, V_{2}$ and $V_{3}$ are vectors tangent to $M$.

Assume that $S$ is some source that emits waves. Given any time $t$, the set of all points of the space to which the wave reaches at time $t$ is called the wavefront at $t$ [3]. The source $S$ might be of any shape. If $S$ is a single point, the wavefront at time $t$ is called the spherical wavefront of radius $t$. The surface tangent to each of these spherical wavefronts is called the envelope of wavefront. There exists some interesting relation between envelope of a wavefront and the next wavefront as follows.

Theorem 2.1. [3] Let $\phi_{p}(t)$ be the wavefront of the point $p$ after time $t$. For every point $q$ of this wavefront, consider the wavefront after time $s$, i.e. $\phi_{q}(s)$. Then, the wavefront of point $p$ after time $s+t, \phi_{p}(s+t)$, will be the envelope of wavefronts $\phi_{q}(s)$, for $q \in \phi_{p}(t)$. 


\section{Providing the paradigm by using the Randers geometry}

In this section we present a paradigm for the spread of wildfire from time 0 to $T$, supposing that $A$ is given as the wavefront at time 0 . In fact, $A$ might be a point, trace of some smooth curve or graph of some smooth surface. Theorem 3.1 provides the paradigm for the case that some time independent constant wind is blowing across the field. In Theorem 3.2, we provide the model for the case that the wind is a time-dependent vector field.

Theorem 3.1. Assume that a fire is spreading across some agricultural field $M$ while the wind $W=$ $\left(0, W_{2}, W_{3}\right)$ is blowing across $M$ and $A$ is the wavefront at time 0 . Then:

(1) Given $p \in A$, the spherical wavefront of some radius $\tau$ and center $p$ is

$$
Q\left(\frac{u}{\tau}, \frac{v-\tau W_{2}}{\tau}, \frac{w-\tau W_{3}}{\tau}\right)+p
$$

where

$$
Q(u, v, w)=\left(\frac{u}{a}\right)^{2}+\left(\frac{v \cos \alpha-w \sin \alpha}{b}\right)^{2}+\left(\frac{v \sin \alpha+w \cos \alpha}{c}\right)^{2}=1
$$

in which $a, b, c$, and $\alpha$ are constant numbers and are determined from the experimental data. Here, we use a right-handed coordinate system and a right-handed rotation through an angle $\alpha$ around $x$-axis.

(2) The equation of each wave ray is $\gamma(t)=p+t V, t \in[0, T]$, such that $p \in A,|V-W|=1$ and $V-W \perp \frac{\perp}{h} A$, where $||=.\sqrt{h(., .)}$ and $h=\frac{1}{2} \operatorname{Hess} Q$.

(3) The wavefront at time $\tau$ is the envelope of $A$.

Proof. Items (1) and (2) are directly resulted from Theorem 3.2 of [4]. To prove item (3), we consider the distance function $\rho: M \rightarrow \mathbb{R}, \rho()=.d(A,$.$) . Since the wavefront at time 0$, that is $A$, coincides with $\rho^{-1}(0)$, by Theorem 6 of [5], the Huygens' envelope principle is satisfied by $\rho^{-1}(0)$ and, therefore, the envelope of $A$ is the wavefront at time $\tau$.

In the next result, we give the paradigm for the case that a time dependent wind $W(t)=$ $\left(W_{1}(t), W_{2}(t), W_{3}(t)\right)$ is blowing across the field and it remains constant at subintervals of time. In other words, assume that $[0, T]$ is the interval of time for which we want to provide the model and $\left\{0=t_{1}, t_{2}, \cdots, t_{n}=T\right\}$ a partition of it. Next, the wind $W(t)$ remains the constant vector $W_{i}=\left(W_{1 i}, W_{2 i}, W_{3 i}\right)$, where $W_{j i}:=W_{j}\left(t_{i}\right)$ for $j=1,2,3$, during each interval $\left[t_{i}, t_{i+1}\right]$, for $i=1, \cdots, n$. The interesting point is that the wind does not have to change smoothly from one interval to the next one.

Theorem 3.2. Assume that a fire is spreading across some agricultural field $M, A$ is the wavefront at time 0 , and some wind $W(t), t \in[0, T]$, is blowing across $M$. If for a given partition $\{0=$ $\left.t_{1}, t_{2}, \cdots, t_{n}=T\right\}$ of $[0, T]$, the wind is some constant vector $W_{i}=\left(W_{1 i}, W_{2 i}, W_{3 i}\right)$, at each interval $\left[t_{i}, t_{i+1}\right], i=1, \cdots, n-1$, then:

(1) Given $p \in A$, the spherical wavefront at time $\tau, \tau \in\left[0, t_{2}\right]$, and center $p$ is

$$
Q_{1}\left(\frac{u}{\tau}, \frac{v-\tau W_{21}}{\tau}, \frac{w-\tau W_{31}}{\tau}\right)+p
$$

where $Q_{1}(u, v, w)$ is given by

$$
Q_{1}(u, v, w)=\left(\frac{u}{a_{1}}\right)^{2}+\left(\frac{v \cos \alpha_{1}-w \sin \alpha_{1}}{b_{1}}\right)^{2}+\left(\frac{v \sin \alpha_{1}+w \cos \alpha_{1}}{c_{1}}\right)^{2}=1,
$$

in which $a_{1}, b_{1}, c_{1}$, and $\alpha_{1}$ are constant numbers and are determined from experimental data. 
(2) Given $p \in A$, the wave ray emanating from $p$ until time $t_{2}$ is $\gamma_{1}(t)=p+t V$, where $V=\left(v_{1}, v_{2}, v_{3}\right)$, such that

$\left\{\begin{array}{l}\left|\left(v_{1}, v_{2}-W_{21}, v_{3}-W_{31}\right)\right|_{1}=1, \\ \left(v_{1}, v_{2}-W_{21}, v_{3}-W_{31}\right) \frac{\perp}{h_{1}} A\end{array}\right.$

where $|\cdot|_{1}=\sqrt{h_{1}(., .)}, h_{1}=\frac{1}{2} \operatorname{Hess} Q_{1}$, and.$\frac{\perp}{h_{1}} A$ means being orthogonal to $A$ with respect to $h_{1}$. Furthermore, the wavefront at time $\tau, \tau \in\left[0, t_{2}\right]$, is the envelope of $A$.

Supposing that the wavefront at time $t_{i}$ is denoted by $\Sigma_{i}, i=1, \cdots, n-1$, we have the following two results:

(3) The spherical wavefront of center $p \in \Sigma_{i}$ and radius $\tau, \tau \in\left(0, t_{i+1}-t_{i}\right]$, is

$$
Q_{i}\left(\frac{u}{\tau}, \frac{v}{\tau}, \frac{w}{\tau}\right)+\tau W_{i}+p
$$

where $Q_{i}(u, v, w)$ is given by

$$
Q_{i}(u, v, w)=\left[\begin{array}{c}
u \\
v \\
w
\end{array}\right]^{T} P^{T} \mathcal{D} P\left[\begin{array}{c}
u \\
v \\
w
\end{array}\right]=1
$$

in which $\mathcal{D}:=\operatorname{diag}\left(\frac{1}{a_{i}^{2}}, \frac{1}{b_{i}^{2}}, \frac{1}{c_{i}^{2}}\right)$ is a diagonal matrix and $P=\mathfrak{R}_{x}\left(\alpha_{i}\right) \mathfrak{R}_{y}\left(\beta_{i}\right) \mathfrak{R}_{z}\left(\theta_{i}\right)$, where

$\mathfrak{R}_{x}\left(\alpha_{i}\right)=\left(\begin{array}{ccc}1 & 0 & 0 \\ 0 & \cos \alpha_{i} & -\sin \alpha_{i} \\ 0 & \sin \alpha_{i} & \cos \alpha_{i}\end{array}\right), \mathfrak{R}_{y}\left(\beta_{i}\right)=\left(\begin{array}{ccc}\cos \beta_{i} & 0 & \sin \beta_{i} \\ 0 & 1 & 0 \\ -\sin \beta_{i} & 0 & \cos \beta_{i}\end{array}\right), \mathfrak{R}_{z}\left(\theta_{i}\right)=\left(\begin{array}{ccc}\cos \theta_{i} & -\sin \theta_{i} & 0 \\ \sin \theta_{i} & \cos \theta_{i} & 0 \\ 0 & 0 & 1\end{array}\right)$,

and $B^{T}$ is the transpose of the matrix $B$. Here $a_{i}, b_{i}, c_{i}, \alpha_{i}, \beta_{i}$, and $\theta_{i}$ are constant numbers and are determined from the experimental data, where $\alpha_{i}, \beta_{i}$, and $\theta_{i}$ are angles of rotation around $x-, y$-, and z-axes, respectively.

(4) The wave ray from time $t_{i}$ to $t_{i+1}, i=2, \cdots, n-1$, is $\gamma_{i}(t)=p+\left(t-t_{i}\right) V, t \in\left[t_{i}, t_{i+1}\right]$, provided that

$\left\{\begin{array}{l}p \in \Sigma_{i}, \\ \left|\left(v_{1}-W_{1 i}, v_{2}-W_{2 i}, v_{3}-W_{3 i}\right)\right|_{i}=1 \\ \left(v_{1}-W_{1 i}, v_{2}-W_{2 i}, v_{3}-W_{3 i}\right) \frac{\perp}{h_{i}} \Sigma_{i},\end{array}\right.$

where $|.|_{i}=\sqrt{h_{i}(., .)}$ and $h_{i}(.,)=.\operatorname{Hess} Q_{i}$. Furthermore, the wavefront at time $\tau \in\left(t_{i}, t_{i+1}\right]$ is the envelope of $\Sigma_{i}$.

Proof. We choose the coordinate system in such a way that, for $t \in\left[0, t_{2}\right]$, the vector $W(t):=W_{1}$ belongs to the $y z$-plane. That is, $W_{1}=\left(0, W_{21}, W_{31}\right)$. Therefore, the items $(1)$ and $(2)$ are direct results from Theorem 3.1 .

To prove items (3) and (4), first, it should be noted once we have the wavefront at time $t_{2}$, that is $\Sigma_{2}$, we can assume that a new propagation starts from $\Sigma_{2}$ which is corresponding to the wind $W\left(t_{2}\right):=W_{2}=\left(W_{12}, W_{22}, W_{32}\right), t \in\left[t_{2}, t_{3}\right)$. Hence, motivated by Theorem 3.1, the spherical wavefront must be some rotated ellipsoid. However, since the wind, $W_{2}$, is not necessarily in $y z$-plane, the ellipsoid must be rotated with some angles $\alpha_{2}, \beta_{2}$, and $\theta_{2}$ around $x-, y$-, and $z$-axis, respectively. Consequently, the spherical wavefront at time $\tau, \tau \in\left(0, t_{3}-t_{2}\right]$, is

$$
Q_{2}\left(\frac{u}{\tau}, \frac{v}{\tau}, \frac{w}{\tau}\right)+\tau W_{2}+p
$$

where $p \in \Sigma_{2}$ and $Q_{2}(u, v, w)$ is given by Eq. (3.6), when $i=2$. Now, considering $\Sigma_{2}$, by item (2) of Theorem 3.1, the wave ray from $p \in \Sigma_{2}$ until time $t_{3}$ is $\gamma_{2}(t)=p+\left(t-t_{2}\right) V, t \in\left[t_{2}, t_{3}\right]$, such that $\left|\left(v_{1}-W_{12}, v_{2}-W_{22}, v_{3}-W_{32}\right)\right|_{2}=1$ and $\left(v_{1}-W_{12}, v_{2}-W_{22}, v_{3}-W_{32}\right) \frac{\perp}{h_{2}} \Sigma_{2}$, where $|\cdot|_{2}=\sqrt{h_{2}(., .)}$ and $h_{2}(.,)=.\operatorname{Hess} Q_{2}$. Also, by item (3) of Theorem 3.1, the wavefront at time $\tau \in\left(t_{2}, t_{3}\right]$ is the 
envelope of $A$.

Now, to conclude the proof of item (3), given the wavefront at time $t_{i}, \Sigma_{i}$, by following the same arguments as above, one shows that the spherical wavefront at time $\tau, \tau \in\left(0, t_{i+1}-t_{i}\right]$ is

$$
Q_{i}\left(\frac{u}{\tau}, \frac{v}{\tau}, \frac{w}{\tau}\right)+\tau W_{i}+p
$$

where $Q_{i}(u, v, w)$ is given by Eq. (3.6).

Finally, to conclude item (4), given the wavefront at time $t_{i}$, one follows the same arguments as those done for the wave rays from time $t_{2}$ to $t_{3}$ and wavefronts at time $\tau, \tau \in\left(t_{2}, t_{3}\right]$.

Remark 3.1. In Theorems 3.1 and 3.2, if the fire starts from a single point, we consider this point as the origin of the coordinate system and replace $A=0$ in expression of theorems.

\section{Example}

Assume that a wildfire is spreading throughout a wheat field $M$ which is a 3 -dimensional smooth manifold and some wind is blowing across $M$. We want to provide a model for the propagation of the wildfire from time 0 to 10 while the wind is a time dependent vector field $W(t)$ such that from time 0 to 5 , the wind is $W=(0,-1 / 3,1 / 6)$ and from time 5 to 10 it is as $W=(1 / 8,0,0)$. We want to provide the model of propagation on the land.

Let $A$ be the path of closed curve

$$
C(s)=\left(\frac{4}{13} \sin s(-\sin s+3), \frac{1}{4} \cos s(\cos s+6), 0\right), s \in[0,2 \pi] .
$$

From Theorem 3.2, we know that the spherical wavefront from time 0 to 5 is the rotated ellipsoid $Q_{1}$ given by Eq. (3.4). Assume that, from the experimental data, we are given the constant numbers corresponding to $Q_{1}$ as follows:

$$
a_{1}=2, b_{1}=1, c_{1}=2, \alpha_{1}=30 .
$$

Here, in Fig. 1, we used the spherical wavefronts and then the Huygens principle to predict the behavior of fire and provide the model of propagation on the land. In this figure (Fig. 1), the waterfronts and the path of some fire's particle from time 0 to 5 are shown.

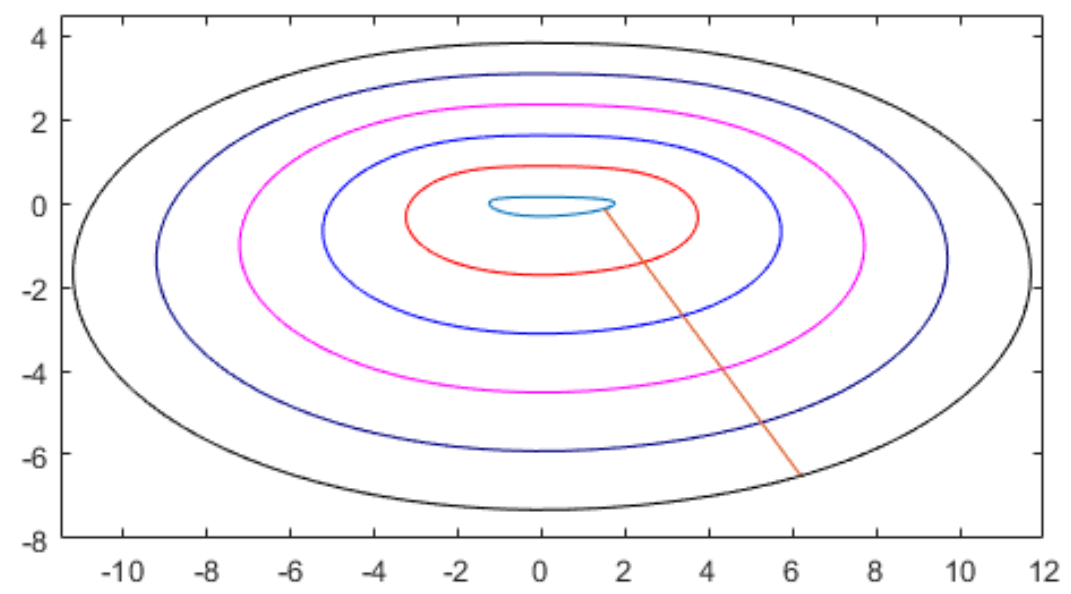

Figure 1: The wavefronts and some wave ray from time 1 to 5 
By the same Theorem, the spherical wavefront from time 5 to 10 is the rotated ellipsoid $Q_{2}$ given by Eq. (3.6). Assume that from the experimental data the constant numbers corresponding to $Q_{2}$ are given as below:

$$
a_{2}=3, b_{2}=1, c_{2}=2, \alpha_{2}=0, \beta_{2}=10, \theta_{2}=0 .
$$

In Fig. 2, we used the spherical wavefronts and then applied the Huygens principle to provide the model of propagation on the land. In this figure (Fig. 2), the wavefronts and the path of some fire's particle from time 0 to 10 are shown.

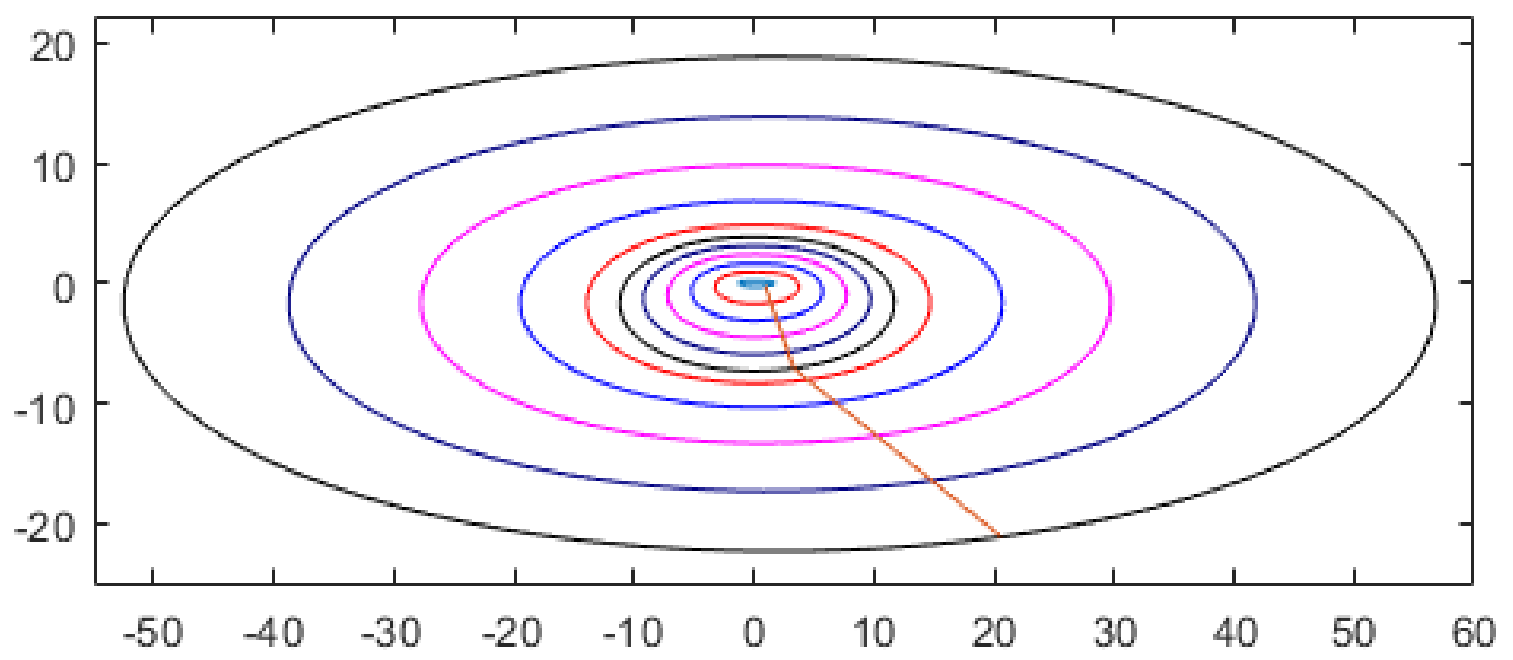

Figure 2: The wavefronts and some wave ray from time 1 to 10

\section{Conclusion}

In this work, for a wildfire spreading in some agricultural land under the presence of wind, some paradigms for the models of spread were presented. In fact, first, it was assumed that the wind is some constant vector field and next the wind was assumed to be some vector field which varies during the time of propagation $[0, T]$; however it remains constant at each interval of time $\left[t_{i}, t_{i+1}\right]$. Here $t_{1}=0, \cdots, t_{n}=T$ is a partition of $[0, T]$. For each case, the equations of spherical wavefronts, wave rays and wavefronts are determined.

\section{References}

[1] Alexandrino, M. M., Alves, B. O., And Dehkordi, H. R. On finsler transnormal functions. Differential Geometry and its Applications 65 (2019), 93-107.

[2] Anderson, D. H., Catchpole, E. A., De Mestre, N. J., and Parkes, T. Modelling the spread of grass fires. Anziam J. 23, 4 (1982), 451-466.

[3] Arnold, V. I. Mathematical methods of classical mechanics, vol. 60. Springer Science \& Business Media, New York, Springer, 2013.

[4] Dehkordi, H. R. Mathematical modeling the wildfire propagation in a Randers space. arXiv preprint arXiv:2012.06692 (2020).

[5] Dehkordi, H. R., And SAA, A. Huygens' envelope principle in Finsler spaces and analogue gravity. Classical Quant. Grav. 36, 8 (2019), 085008.

[6] Fuster, A., And Pabst, C. Finsler p p-waves. Phys. Rev. D 94, 10 (2016), 104072.

[7] Giambò, R., Giannoni, F., and Piccione, P. Genericity of nondegeneracy for light rays in stationary spacetimes. Comm. Math. Phys. 287, 3 (2009), 903-923. 
[8] Glasa, J., And Halada, L. On mathematical foundations of elliptical forest fire spread model. Chapter 12 (2009), 315-333

[9] Guelpa, E., Sciacovelli, A., Verda, V., And Ascoli, D. Faster prediction of wildfire behaviour by physical models through application of proper orthogonal decomposition. IJWF 25, 11 (2016), 1181-1192.

[10] Markvorsen, S. A Finsler geodesic spray paradigm for wildfire spread modelling. Nonlinear Anal. Real World Appl. 28 (2016), 208-228.

[11] Papadopoulos, G. D., and Pavlidou, F. N. A comparative review on wildfire simulators. IEEE Syst J. 5, 2 (2011), 233-243.

[12] Wiedinmyer, C., And NefF, J. C. Estimates of $C O^{2}$ from fires in the united states: implications for carbon management. Carbon Balance Manag. 2, 1 (2007), 10. 